\title{
Bounds for Incidence Energy of Some Graphs
}

\author{
Weizhong Wang ${ }^{1,2}$ and Dong Yang ${ }^{2}$ \\ ${ }^{1}$ Department of Mathematics, Lanzhou Jiaotong University, Lanzhou 730070, China \\ ${ }^{2}$ Department of Mathematics, Lanzhou University, Lanzhou 730000, China
}

Correspondence should be addressed to Weizhong Wang; jdslxywwz@163.com

Received 23 April 2013; Accepted 2 July 2013

Academic Editor: Magdy A. Ezzat

Copyright (C) 2013 W. Wang and D. Yang. This is an open access article distributed under the Creative Commons Attribution License, which permits unrestricted use, distribution, and reproduction in any medium, provided the original work is properly cited.

Let $G$ be a simple graph. The incidence energy ( $I E$ for short) of $G$ is defined as the sum of the singular values of the incidence matrix. In this paper, a new upper bound for $I E$ of graphs in terms of the maximum degree is given. Meanwhile, bounds for $I E$ of the line graph of a semiregular graph and the paraline graph of a regular graph are obtained.

\section{Introduction}

Let $G$ be a finite, simple, and undirected graph with $n$ vertices. The matrix $L(G)=D(G)-A(G)\left(\right.$ resp., $L^{+}(G)=D(G)+A(G)$ ) is called the Laplacian matrix (resp., signless Laplacian matrix [1-4]) of $G$, where $A(G)$ is the adjacency matrix and $D(G)$ is the diagonal matrix of the vertex degrees. (For details on Laplacian matrix, see $[5,6]$.) Since $A(G), L(G)$ and $L^{+}(G)$ are all real symmetric matrices, their eigenvalues are real numbers. So, we can assume that $\lambda_{1}(G) \geq \lambda_{2}(G) \geq \cdots \geq$ $\lambda_{n}(G)$ (resp., $\mu_{1}(G) \geq \mu_{2}(G) \geq \cdots \geq \mu_{n}(G), \mu_{1}^{+}(G) \geq \mu_{2}^{+}(G) \geq$ $\cdots \geq \mu_{n}^{+}(G)$ ) are the adjacency (resp., Laplacian, signless Laplacian) eigenvalues of $G$. It follows from the Geršgorin disc theorem that $L(G)$ and $L^{+}(G)$ are semidefinite. Therefore, all Laplacian (resp., signless Laplacian) eigenvalues of $G$ are nonnegative. If the graph $G$ is connected, then $\mu_{i}(G)>0$ for $i=1,2, \ldots, n-1$ and $\mu_{n}(G)=0$ [6]. If $G$ is a connected nonbipartite graph, then $\mu_{i}^{+}(G)>0$ for $i=1,2, \ldots, n$ [1].

One of the most remarkable chemical applications of graph theory is based on the close correspondence between the graph eigenvalues and the molecular orbital energy levels of $\pi$-electrons in conjugated hydrocarbons. For the Hüchkel molecular orbital approximation, the total $\pi$-electron energy in conjugated hydrocarbons is given by the sum of absolute values of the eigenvalues corresponding to the molecular graph $G$ in which the maximum degree is not more than four in general. The energy of $G$ was defined by Gutman in [7] as

$$
E(G)=\sum_{i=1}^{n}\left|\lambda_{i}(G)\right|
$$

Research on graph energy is nowadays very active, as seen from the recent papers [8-15], monograph [16], and the references quoted therein.

The singular values of a real matrix (not necessarily square) $M$ are the square roots of the eigenvalues of the matrix $M M^{t}$, where $M^{t}$ denotes the transpose of $M$. Recently, Nikiforov [17] extended the concept of graph energy to any matrix $M$ by defining the energy $E(M)$ to be the sum of singular values of $M$. Obviously, $E(G)=E(A(G))$.

Let $I(G)$ be the (vertex-edge) incidence matrix of the graph $G$. For a graph $G$ with vertex set $\left\{v_{1}, v_{2}, \ldots, v_{n}\right\}$ and edge set $\left\{e_{1}, e_{2}, \ldots, e_{m}\right\}$, the $(i, j)$-entry of $I(G)$ is 0 if $v_{i}$ is not incident with $e_{j}$ and 1 if $v_{i}$ is incident with $e_{j}$. Jooyandeh et al. [18] introduced the incidence energy IE of $G$, which is defined as the sum of the singular values of the incidence matrix of $G$. Gutman et al. [19] showed that

$$
\mathrm{IE}=\mathrm{IE}(G)=\sum_{i=1}^{n} \sqrt{\mu_{i}^{+}(G)} .
$$

Some basic properties of IE may be found in [18-20]. 
A line graph is a classical unary operation of graphs with finite number and infinite number of vertices. Its basic properties can be found in any text book on graph theory (see, e.g., [21-23]). Recently, several papers on line graph have been published [20, 24-27]. For example, Gao et al. [24] established a formula and lower bounds for the Kirchhoff index of the line graph of a regular graph. Bounds for Laplacian-energylike invariant (LEL for short) of the line graph of a regular graph $G$ are obtained in [26]. For details on LEL, see the comprehensive survey [28].

From (2), one can immediately get the incidence energy of a graph by computing the signless Laplacian eigenvalues of the graph. However, even for special graphs, it is still complicated to find the signless Laplacian eigenvalues of them. Hence, it makes sense to establish lower and upper bounds to estimate the invariant for some classes of graphs. Zhou [29] obtained the upper bounds for the incidence energy using the first Zagreb index. Gutman et al. [20] gave several lower and upper bounds for IE. In particular, an upper bound for IE of the line graph of a regular graph was established in [20].

In this paper, we continue to study the bounds for IE of graphs. In Section 2, we give a new upper bound for IE of graphs in terms of the maximum degree. Bounds for IE of the line graph of a semiregular graph and the paraline graph of a regular graph are obtained in Section 3.

\section{A New Upper Bound for $I E$}

In this section, we will give a new upper bound for IE of a nonempty graph. The following fundamental properties of the IE were established in [18].

Lemma 1 (see [18]). Let $G$ be a graph with $n$ vertices and $m$ edges. Then

(i) $\operatorname{IE}(G) \geq 0$, and equality holds if and only if $m=0$;

(ii) if $G_{1}, \ldots, G_{p}$ are all components of $G$, then $\operatorname{IE}(G)=$ $\sum_{i}^{p} \operatorname{IE}\left(G_{i}\right)$.

From Lemma 1(ii), when we study the incidence energy of a graph $G$, we may assume that $G$ is connected.

The following lemma will be used later.

Lemma 2 (see [3]). Let $G$ be a connected graph without vertices of degree 1 and the maximum degree $\Delta$. Then, $\mu_{1}^{+} \geq$ $1+\Delta+(1 /(\Delta-1))$; the equality holds if and only if $G$ is a cycle.

Denote by $C_{n}$ the cycle with $n$ vertices.

Theorem 3. Let $G$ be a connected graph without vertices of degree 1 and the maximum degree $\Delta$. Then

$$
\begin{aligned}
\operatorname{IE}(G) \leq & \sqrt{1+\Delta+\frac{1}{\Delta-1}} \\
& +\sqrt{(n-1)\left(2 m-1-\Delta-\frac{1}{\Delta-1}\right)}
\end{aligned}
$$

the equality holds if and only if $G \cong C_{3}$.
Proof. Note that $\sum_{i=2}^{n} \mu_{i}^{+}=2 m-\mu_{1}^{+}$. By the Cauchy-Schwarz inequality

$$
\operatorname{IE}(G) \leq \sqrt{\mu_{1}^{+}}+\sqrt{(n-1)\left(2 m-\mu_{1}^{+}\right)} .
$$

The equality holds if and only if $\mu_{2}^{+}=\cdots=\mu_{n}^{+}$.

We consider the function

$$
f(x)=\sqrt{x}+\sqrt{(n-1)(2 m-x)}, \quad x>0 .
$$

Then

$$
f^{\prime}(x)=\frac{1}{2 \sqrt{x}}+\frac{-n+1}{2 \sqrt{(n-1)(2 m-x)}} .
$$

It is easily seen that the function $f(x)$ is decreasing for $x>$ $2 m / n$. By Lemma 2 ,

$$
\frac{2 m}{n} \leq \Delta<1+\Delta<1+\Delta+\frac{1}{\Delta-1} \leq \mu_{1}^{+} .
$$

Inequality (3) follows now from the monotonicity of $f(x)$ and (4).

Equality in (3) holds if and only if $G$ is a cycle. It is wellknown [4] that the signless Laplacian eigenvalues of $C_{n}$ are

$$
2+2 \cos \frac{2 \pi}{n} j \quad(j=0,1, \ldots, n-1) .
$$

If $G \cong C_{3}$, then we may verify directly that the equality in (3) holds.

Conversely, if the equality in (3) holds, then

$$
\mu_{1}^{+}=1+\Delta+\frac{1}{\Delta-1}, \quad \mu_{2}^{+}=\cdots=\mu_{n}^{+} .
$$

It follows from Lemma 2 that $G$ is a cycle. Note that $G$ has at most two distinct signless Laplacian eigenvalues. By virtue of (8), we now conclude that $G$ is a triangle.

Recall from [20] that an upper bound for IE was given as follows.

Lemma 4 (see [20]). Let $G$ be a connected graph with $n \geq 3$ vertices and $m$ edges. Then

$$
\operatorname{IE}(G)<\sqrt{1+\Delta}+\sqrt{(n-1)(2 m-1-\Delta)} .
$$

Remark 5. It should be pointed out that, for a connected graph without vertices of degree 1 , the bound in (3) is better than the bound in (10). Indeed, it is easily seen that the bound in (3) is $f(1+\Delta+(1 /(\Delta-1)))$, but the bound in (10) is $f(1+\Delta)$. Note that

$$
\frac{2 m}{n} \leq \Delta<1+\Delta<1+\Delta+\frac{1}{\Delta-1} \leq \mu_{1}^{+} .
$$

It follows from the monotonicity of $f(t)$ that

$$
f\left(1+\Delta+\frac{1}{\Delta-1}\right)<f(1+\Delta) .
$$

That is,

$$
\begin{aligned}
\sqrt{1+\Delta+\frac{1}{\Delta-1}}+\sqrt{(n-1)\left(2 m-1-\Delta-\frac{1}{\Delta-1}\right)} \\
<\sqrt{1+\Delta}+\sqrt{(n-1)(2 m-1-\Delta)} .
\end{aligned}
$$




\section{Bounds for $I E$ of Line Graphs of Semiregular Graphs}

In this section, we will investigate the IE of the line graph of an $(r, s)$-semiregular graph and the paraline graph of an $r$ regular graph.

We first consider the case for line graph. The line graph $\mathscr{L}(G)$ of a graph $G$ is the graph whose vertex set is in oneto-one correspondence with the set of edges of $G$ where two vertices of $\mathscr{L}(G)$ are adjacent if and only if the corresponding edges in $G$ have a vertex in common. For instance, the line graph of a star $S_{n}$ on $n$ vertices is a complete graph $K_{n-1}$ on $n-1$ vertices.

The following result is well known [30].

Lemma 6 (see [30]). Let $A$ be a matrix. Then, the matrices $A A^{T}$ and $A^{T} A$ have the same nonzero eigenvalues.

A bipartite graph $G$ with a bipartition $V(G)=U \cup W$ is called an $(r, s)$-semiregular graph if all vertices in $U$ have degree $r$ and all vertices in $W$ have degree $s$. Denote by $\mu_{G}^{+}(x)$ the signless Laplacian polynomial $\operatorname{det}\left(x I-L^{+}(G)\right)$ of $G$. For an $(r, s)$-semiregular graph $G$, we can establish the following relationship between $\mu_{G}^{+}(x)$ and $\mu_{\mathscr{L}(G)}^{+}(x)$.

Lemma 7. Let $G$ be an $(r, s)$-semiregular graph with $n$ vertices. Then

$$
\mu_{\mathscr{L}(G)}^{+}(\mu)=(\mu-(r+s-4))^{m-n} \mu_{G}^{+}(\mu-(r+s-4)),
$$

where $\mathscr{L}(G)$ is the line graph of $G$ and $m$ is the number of edges of $G$.

Proof. Let $I(G)$ be the (vertex-edge) incidence matrix of a graph $G$. Then

$$
I(G) I(G)^{T}=L^{+}(G), \quad I(G)^{T} I(G)=2 I_{m}+A(\mathscr{L}(G)),
$$

where $I_{m}$ stands for the unit matrix of order $m$.

Note that if $G$ is an $(r, s)$-semiregular graph, then the line graph of $G$ is $(r+s-2)$-regular graph. Thus

$$
L^{+}(\mathscr{L}(G))=(r+s-2) I_{m}+A(\mathscr{L}(G)) .
$$

Combine (15) with (16), we have

$$
L^{+}(\mathscr{L}(G))-(r+s-4) I_{m}=I(G)^{T} I(G) .
$$

It follows from Lemma 6, (15), and (17) that $L^{+}(G)$ and $L^{+}(\mathscr{L}(G))-(r+s-4) I_{m}$ have the same nonzero eigenvalues. Note that the difference between the dimensions of $L^{+}(\mathscr{L}(G))$ and $L^{+}(G)$ is $m-n$. The proof is finished by the fact that the leading coefficient of the characteristic polynomial is equal to one. are

By Lemma 7, the signless Laplacian eigenvalues of $\mathscr{L}(G)$

$$
\left(\begin{array}{ccccc}
r+s-4 & r+s-4+\mu_{1}^{+} & r+s-4+\mu_{2}^{+} & \cdots & r+s-4+\mu_{n}^{+} \\
m-n & 1 & 1 & \cdots & 1
\end{array}\right)
$$

where $\mu_{1}^{+} \geq \mu_{2}^{+} \geq \cdots \geq \mu_{n}^{+}$are the signless Laplacian eigenvalues of $G$.

Theorem 8. Let $G$ be an $(r, s)$-semiregular graph with $n$ vertices. Then

$$
\begin{aligned}
\operatorname{IE}(\mathscr{L}(G)) \leq & \left(\frac{n r s}{r+s}-n+1\right) \sqrt{r+s-4}+\sqrt{2(r+s)-4} \\
& +\sqrt{(n-2)\left[(n-3)(r+s)-4(n-2)+\frac{2 n r s}{r+s}\right]},
\end{aligned}
$$

the equality holds if and only if $G \cong K_{1, n-1}$, or $n$ is even and $G \cong K_{(n / 2),(n / 2)}$ with $n \geq 4$.

Proof. Let $m$ be the number of edges of $G$. Then, $m=n r s /(r+$ $s)$. Note that $\mu_{1}^{+}(G)=r+s$. It follows from (2) and (18) that

$$
\begin{aligned}
\operatorname{IE}(\mathscr{L}(G))= & (m-n) \sqrt{r+s-4}+\sum_{i=1}^{n} \sqrt{r+s-4+\mu_{i}^{+}} \\
= & (m-n) \sqrt{r+s-4}+\sqrt{2(r+s)-4} \\
& +\sum_{i=2}^{n} \sqrt{r+s-4+\mu_{i}^{+}} .
\end{aligned}
$$

Note also that $\mu_{n}^{+}=0, \sum_{i=1}^{n} \mu_{i}^{+}=2 m$. By the Cauchy-Schwarz inequality, we have

$$
\begin{aligned}
\sum_{i=2}^{n} \sqrt{r+s-4+\mu_{i}^{+}} & \sqrt{r+s-4}+\sum_{i=2}^{n-1} \sqrt{r+s-4+\mu_{i}^{+}} \\
= & \sqrt{r+s-4}+\sqrt{(n-2) \sum_{i=2}^{n-1}\left(r+s-4+\mu_{i}^{+}\right)} \\
= & \sqrt{r+s-4} \\
& +\sqrt{(n-2)\left[(n-2)(r+s)-4(n-2)+\left(2 m-\mu_{1}^{+}\right)\right]} \\
= & \sqrt{r+s-4} \\
& +\sqrt{(n-2)\left[(n-3)(r+s)-4(n-2)+\frac{2 n r s}{r+s}\right]} .
\end{aligned}
$$

Inequality (19) follows now from (20) and (21).

Clearly, equality in (19) holds if and only if $\mu_{2}^{+}=\cdots=$ $\mu_{n-1}^{+}$. Suppose that $\mu_{2}^{+}=\cdots=\mu_{n-1}^{+}$. Then, the number of distinct signless Laplacian eigenvalues of $G$ is at most 3. From the result, "the number of distinct signless Laplacian eigenvalues of a connected graphs with diameter $d$ is at least $d+1$ [1]," we know that the diameter of $G$ is at most 2 . Note that $G$ is bipartite graph. If the diameter of $G$ is 1 , then $G$ must be $K_{2}$. If the diameter of $G$ is 2 , then $G$ is a complete bipartite graph $K_{p, q}$ with exactly 3 distinct signless Laplacian 
eigenvalues. It is well known that the signless Laplacian eigenvalues of $K_{p, q}$ are $p+q, p^{q-1}, q^{p-1}$, and 0 . Thus, $G \cong$ $K_{1, n-1}$, or $n$ is even and $G \cong K_{(n / 2),(n / 2)}$ with $n \geq 4$.

Conversely, if $G \cong K_{1, n-1}$, then $\mathscr{L}(G) \cong K_{n-1}$. Note that the signless Laplacian spectrum of complete graph $K_{t}[4]$ is

$$
\left(\begin{array}{cc}
t-2 & 2 t-2 \\
t-1 & 1
\end{array}\right)
$$

It follows from (2) and (22) that

$$
\operatorname{IE}(\mathscr{L}(G))=\operatorname{IE}\left(K_{n-1}\right)=\sqrt{2 n-4}+(n-2) \sqrt{n-3}
$$

That is, the left-hand side of (19) is equal to $\sqrt{2 n-4}+$ $(\mathrm{n}-2) \sqrt{n-3}$. In this case, $r=n-1$ and $s=1$. It is easy to check that the right-hand side of (19) is also equal to $\sqrt{2 n-4}+(n-$ 2) $\sqrt{n-3}$. If $G \cong K_{(n / 2),(n / 2)}$, then $\mathscr{L}(G) \cong L_{2}(n / 2)$, where $n \geq 4$ is even. Note also that the signless Laplacian spectrum of lattice graph $L_{2}(p)$ [31] is

$$
\left(\begin{array}{ccc}
2 p-4 & 4 p-4 & 3 p-4 \\
(p-1)^{2} & 1 & 2 p-2
\end{array}\right)
$$

Similarly, it follows from (2) and (24) that the left-hand side of (19) is equal to $(n / 2-1)^{2} \sqrt{n-4}+\sqrt{2 n-4}+$ $(n-2) \sqrt{3 n / 2-4}$. Note that $K_{(n / 2),(n / 2)}$ is an $(n / 2, n / 2)$ semiregular graph. Substituting $r=s=n / 2$ into (19), we get the right-hand side of (19) is still equal to $(n / 2-1)^{2} \sqrt{n-4}+$ $\sqrt{2 n-4}+(n-2) \sqrt{3 n / 2-4}$.

Hence, we complete the proof of Theorem 8 .

Now, we consider the case for paraline graph. Let $G$ be a simple graph. A paraline graph, denoted by $C(G)$, is defined as a line graph of the subdivision graph $s(G)$ (the subdivision graph $s(G)$ of a graph $G$ is the graph obtained from $G$ by inserting a vertex to every edge of $G$ ) of $G$ (e.g., see Figure 1). The concept of the paraline graph (or clique-inserted graph [32]) of a graph was first introduced in [25], where the author obtained the spectrum of the paraline graph of a regular graph $G$ with infinite number of vertices in terms of the spectrum of $G$.
Remark 9. The subdivision graph of an $r$-regular graph is $(r, 2)$-semiregular. Hence, the paraline graph of an $r$-regular graph is the line graph of an $(r, 2)$-semiregular graph.

The following result is well known $[5,6]$.

Lemma 10. The spectra of $L(G)$ and $L^{+}(G)$ coincide if and only if the graph $G$ is bipartite. G.

Let $\mu_{G}(x)$ be the Laplacian polynomial $\operatorname{det}(x I-L(G))$ of

Corollary 11. Let $G$ be an $r$-regular graph with $n$ vertices. Then

$$
\begin{aligned}
\mu_{C(G)}^{+}(\mu)=( & -1)^{n r}((r-\mu)(\mu-r+2))^{n r / 2-n} \\
& \times \mu_{G}((2 r-\mu)(\mu-r+2)) .
\end{aligned}
$$

Proof. Note that $s(G)$ is a $(2, r)$-semiregular graph with $n+$ $n r / 2$ vertices and $n r$ edges. By Lemma 7, we obtain

$$
\mu_{C(G)}^{+}(\mu)=(\mu-r+2)^{m-n} \mu_{s(G)}^{+}(\mu-r+2) .
$$

It is shown in [33] that

$$
\mu_{s(G)}(\mu)=(-1)^{m}(2-\mu)^{m-n} \mu_{G}(\mu(r+2-\mu)) .
$$

It follows from (27) and Lemma 10 that

$$
\mu_{s(G)}^{+}(\mu)=(-1)^{m}(2-\mu)^{m-n} \mu_{G}(\mu(r+2-\mu)) .
$$

Combining (26) with (28), we have

$$
\begin{aligned}
\mu_{C(G)}^{+}(\mu)= & (-1)^{n r}((r-\mu)(\mu-r+2))^{n r / 2-n} \\
& \times \mu_{G}((2 r-\mu)(\mu-r+2)) .
\end{aligned}
$$

It follows from Corollary 11 that the signless Laplacian spectrum of $C(G)$ is

$$
\left(\begin{array}{ccccccc}
r & r-2 & \mu_{1}^{+}(C(G)) & \mu_{1}^{\prime+}(C(G)) & \cdots & \mu_{n}^{+}(C(G)) & \mu_{n}^{\prime+}(C(G)) \\
\frac{n(r-2)}{2} & \frac{n(r-2)}{2} & 1 & 1 & \cdots & 1 & 1
\end{array}\right)
$$

where

$$
\begin{aligned}
\mu_{i}^{+}(C(G)) & =\frac{3 r-2+\sqrt{(r+2)^{2}-4 \mu_{i}}}{2}, \\
\mu_{i}^{\prime+}(C(G)) & =\frac{3 r-2-\sqrt{(r+2)^{2}-4 \mu_{i}}}{2},
\end{aligned}
$$

Theorem 12. Let $G$ be a connected $r$-regular graph with $n$ vertices. Then

$$
\begin{aligned}
\operatorname{IE}(C(G)) \leq & \left(\frac{n r}{2}+\sqrt{2}-1\right) \sqrt{r}+\sqrt{2}(n-1) \sqrt{r-1} \\
& +\left(\frac{n r}{2}-n+1\right) \sqrt{r-2}
\end{aligned}
$$

and $\mu_{i}, i=1,2, \ldots, n$ are the Laplacian eigenvalues of $G$. 


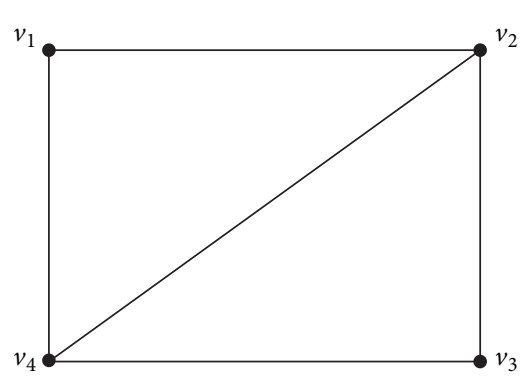

(a)

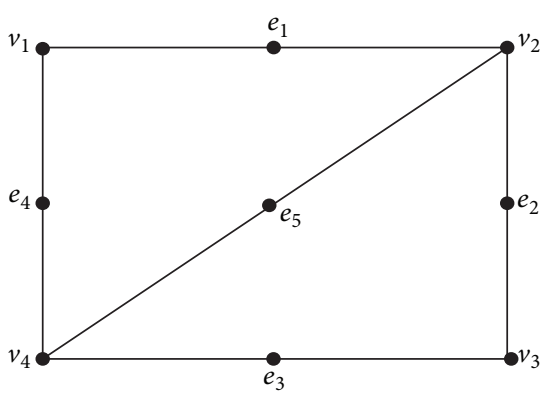

(b)

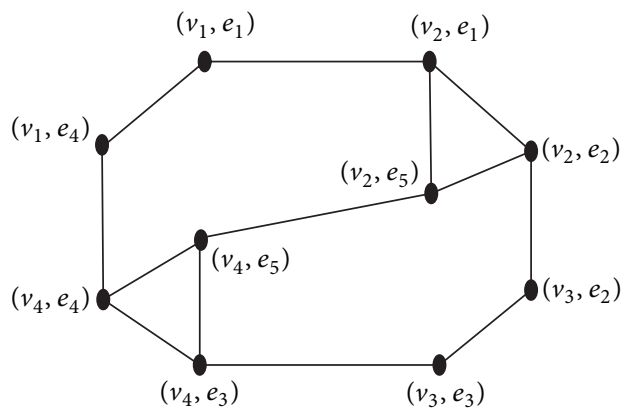

(c)

Figure 1: (a) The graph $G$. (b) The graph $s(G)$. (c) The graph $C(G)$.

Proof. If $r=1$, then $G \cong K_{2}$. Note that $C\left(K_{2}\right) \cong K_{2}$. It is easily seen that

$$
\operatorname{IE}\left(C\left(K_{2}\right)\right)=\sqrt{2} \text {. }
$$

In this case, $r=1$ and $n=2$. Hence

$$
\begin{gathered}
\left(\frac{n r}{2}+\sqrt{2}-1\right) \sqrt{r}+\sqrt{2}(n-1) \sqrt{r-1} \\
+\left(\frac{n r}{2}-n+1\right) \sqrt{r-2}=\sqrt{2}
\end{gathered}
$$

Suppose now that $r \geq 2$. For convenience, let $m=n r / 2$ be the edges of $G$. Note that $G$ is an $r$-regular graph. It follows from the definition of paraline graph that $C(G)$ is still an $r$ regular graph. Note also that $\mu_{i}(G)=r-\lambda_{n-i+1}(G), i=$ $1,2, \ldots, n$ and $\lambda_{1}(G)=r$. Then by (2) and (30), we have

$$
\begin{aligned}
& \operatorname{IE}(C(G)) \\
& =(m-n) \sqrt{r}+(m-n) \sqrt{r-2} \\
& +\sum_{i=1}^{n} \sqrt{\frac{3 r-2+\sqrt{r^{2}+4\left(\lambda_{i}+1\right)}}{2}} \\
& +\sum_{i=1}^{n} \sqrt{\frac{3 r-2-\sqrt{r^{2}+4\left(\lambda_{i}+1\right)}}{2}} \\
& =(m-n+\sqrt{2}) \sqrt{r}+(m-n+1) \sqrt{r-2} \\
& +\sum_{i=2}^{n}\left(\sqrt{\frac{3 r-2+\sqrt{r^{2}+4\left(\lambda_{i}+1\right)}}{2}}\right. \\
& \left.+\sqrt{\frac{3 r-2-\sqrt{r^{2}+4\left(\lambda_{i}+1\right)}}{2}}\right) .
\end{aligned}
$$

Note that $-r \leq \lambda_{i}(G)<r, i=2,3, \ldots, n$, by the PerronFrobenius theorem. Consider the function

$$
\begin{aligned}
g(t)= & \sqrt{\frac{3 r-2+\sqrt{r^{2}+4 t+4}}{2}} \\
& +\sqrt{\frac{3 r-2-\sqrt{r^{2}+4 t+4}}{2}}, \quad-r \leq t<r .
\end{aligned}
$$

The derivative function of $g(t)$ is

$$
\begin{aligned}
g^{\prime}(t)= & \left(\sqrt{\frac{3 r-2-\sqrt{r^{2}+4 t+4}}{2}}\right. \\
& \left.-\sqrt{\frac{3 r-2+\sqrt{r^{2}+4 t+4}}{2}}\right) \\
& \times\left(2 \sqrt{\left(2 r^{2}-(3 r+t)\right)\left(r^{2}+4 t+4\right)}\right)^{-1}, \quad-r \leq t<r .
\end{aligned}
$$

It is clear that $g(t)$ is decreasing for $-r \leq t<r$. Therefore

$$
\begin{aligned}
\operatorname{IE}(C(G)) \leq & (m-n+\sqrt{2}) \sqrt{r} \\
& +(m-n+1) \sqrt{r-2}+\sum_{i=2}^{n} g(-r) \\
= & \left(\frac{n r}{2}+\sqrt{2}-1\right) \sqrt{r}+\sqrt{2}(n-1) \sqrt{r-1} \\
& +\left(\frac{n r}{2}-n+1\right) \sqrt{r-2} .
\end{aligned}
$$

That is, $I E(C(G))=(m-n+\sqrt{2}) \sqrt{r}+(m-n+1) \sqrt{r-2}+$ $\sum_{i=2}^{n} g(-r)$ if and only if $G$ is regular graph and $\lambda_{1}(G)=$ $r, \lambda_{2}(G)=\cdots=\lambda_{n}(G)=-r$. It follows that $G$ is a regular graph with two distinct adjacency eigenvalues $r$ and $-r$ with multiplicities 1 and $n-1$, respectively. Then by Proposition 1.3.3 [31], $G$ must be a complete graph. Note that the sum of the adjacency eigenvalues of $G$ is equal to zero; that is, $r+(n-1)(-r)=0$. It follows that $G$ is a complete graph with two vertices; that is, $G \cong K_{2}$. This is impossible since $r \geq 2$.

Summing up, we complete the proof. 
Theorem 13. Let $G$ be an $r$-regulargraph with $n$ vertices, $r \geq 2$. Then

$$
\operatorname{IE}(C(G))>\frac{n(r+2 \sqrt{2}-2)}{2} \sqrt{r}+\frac{n r}{2} \sqrt{r-2} .
$$

Proof. From the proof of Theorem 12, we know that the function $g(t)$ is decreasing for $-r \leq t<r$. Therefore

$$
\begin{aligned}
\operatorname{IE}(C(G))> & (m-n+\sqrt{2}) \sqrt{r} \\
& +(m-n+1) \sqrt{r-2}+\sum_{i=2}^{n} g(r) \\
& =\frac{n(r+2 \sqrt{2}-2)}{2} \sqrt{r}+\frac{n r}{2} \sqrt{r-2} .
\end{aligned}
$$

\section{Acknowledgments}

The authors are grateful to the anonymous referees for many valuable comments and suggestions, which led to great improvements of the original paper. This research was partially supported by the National Natural Science Foundation of China (no. 11201201) and the Fundamental Research Funds for the Central Universities (no. lzujbky2012-16).

\section{References}

[1] D. Cvetković, P. Rowlinson, and S. K. Simić, "Signless Laplacians of finite graphs," Linear Algebra and its Applications, vol. 423, no. 1, pp. 155-171, 2007.

[2] D. Cvetković and S. K. Simić, "Towards a spectral theory of graphs based on the signless Laplacian. III," Applicable Analysis and Discrete Mathematics, vol. 4, no. 1, pp. 156-166, 2010.

[3] D. Cvetković and S. K. Simić, "Towards a spectral theory of graphs based on the signless Laplacian. II," Linear Algebra and its Applications, vol. 432, no. 9, pp. 2257-2272, 2010.

[4] D. Cvetković and S. K. Simić, "Towards a spectral theory of graphs based on the signless Laplacian. I," Institut Mathématique. Publications, vol. 85, no. 99, pp. 19-33, 2009.

[5] R. Merris, "A survey of graph Laplacians," Linear and Multilinear Algebra, vol. 39, no. 1-2, pp. 19-31, 1995.

[6] R. Merris, "Laplacian matrices of graphs: a survey," Linear Algebra and its Applications, vol. 197-198, pp. 143-176, 1994.

[7] I. Gutman, “The energy of a graph,” Graz. Forschungszentrum. Mathematisch-Statistische Sektion. Berichte, vol. 103, no. 100105, pp. 1-22, 1978.

[8] I. Gutman, "The energy of a graph: old and new results," in Algebraic Combinatorics and Applications, A. Betten, A. Kohnert, and R. Laue, Eds., pp. 196-211, Springer, Berlin, Germany, 2001.

[9] I. Gutman, X. Li, and J. Zhang, "Graph energy," in Analysis of Complex Networks. From Biology to Linguistics, M. Dehmer and F. Emmert-Streib, Eds., pp. 145-174, Wiley-VCH, Weinheim, Germany, 2009.

[10] H. Hua and M. Wang, "Unicyclic graphs with given number of pendent vertices and minimal energy," Linear Algebra and its Applications, vol. 426, no. 2-3, pp. 478-489, 2007.
[11] B. Huo, S. Ji, X. Li, and Y. Shi, "Solution to a conjecture on the maximal energy of bipartite bicyclic graphs," Linear Algebra and its Applications, vol. 435, no. 4, pp. 804-810, 2011.

[12] S. Li, X. Li, and Z. Zhu, "On tricyclic graphs with minimal energy," MATCH. Communications in Mathematical and in Computer Chemistry, vol. 59, no. 2, pp. 397-419, 2008.

[13] Y. Liu, "Some results on energy of unicyclic graphs with $n$ vertices," Journal of Mathematical Chemistry, vol. 47, no. 1, pp. $1-10,2010$.

[14] J. Rada and A. Tineo, "Upper and lower bounds for the energy of bipartite graphs," Journal of Mathematical Analysis and Applications, vol. 289, no. 2, pp. 446-455, 2004.

[15] J. Zhu, "Minimal energies of trees with given parameters," Linear Algebra and its Applications, vol. 436, no. 9, pp. 3120-3131, 2012.

[16] X. Li, Y. Shi, and I. Gutman, Graph Energy, Springer, New York, NY, USA, 2012.

[17] V. Nikiforov, "The energy of graphs and matrices," Journal of Mathematical Analysis and Applications, vol. 326, no. 2, pp. 1472-1475, 2007.

[18] M. Jooyandeh, D. Kiani, and M. Mirzakhah, "Incidence energy of a graph," MATCH. Communications in Mathematical and in Computer Chemistry, vol. 62, no. 3, pp. 561-572, 2009.

[19] I. Gutman, D. Kiani, and M. Mirzakhah, "On incidence energy of graphs," MATCH. Communications in Mathematical and in Computer Chemistry, vol. 62, no. 3, pp. 573-580, 2009.

[20] I. Gutman, D. Kiani, M. Mirzakhah, and B. Zhou, "On incidence energy of a graph," Linear Algebra and its Applications, vol. 431, no. 8, pp. 1223-1233, 2009.

[21] J. A. Bondy and U. S. R. Murty, Graph Theory with Applications, American Elsevier, New York, NY, USA, 1976.

[22] D. M. Cvetković, M. Doob, and H. Sachs, Spectra of Graphs. Theory and Application, Academic Press, New York, NY, USA, 1980.

[23] C. Godsil and G. Royle, Algebraic Graph Theory, Springer, New York, NY, USA, 2001.

[24] X. Gao, Y. Luo, and W. Liu, "Kirchhoff index in line, subdivision and total graphs of a regular graph," Discrete Applied Mathematics, vol. 160, no. 4-5, pp. 560-565, 2012.

[25] T. Shirai, “The spectrum of infinite regular line graphs," Transactions of the American Mathematical Society, vol. 352, no. 1, pp. 115-132, 2000.

[26] W. Wang and Y. Luo, "On Laplacian-energy-like invariant of a graph," Linear Algebra and its Applications, vol. 437, no. 2, pp. 713-721, 2012.

[27] W. Yan, Y.-N. Yeh, and F. Zhang, "The asymptotic behavior of some indices of iterated line graphs of regular graphs," Discrete Applied Mathematics, vol. 160, no. 7-8, pp. 1232-1239, 2012.

[28] B. Liu, Y. Huang, and Z. You, "A survey on the Laplacian-energylike invariant," MATCH. Communications in Mathematical and in Computer Chemistry, vol. 66, no. 3, pp. 713-730, 2011.

[29] B. Zhou, "More upper bounds for the incidence energy," MATCH. Communications in Mathematical and in Computer Chemistry, vol. 64, no. 1, pp. 123-128, 2010.

[30] R. A. Horn and C. R. Johnson, Matrix Analysis, Cambridge University Press, Cambridge, UK, 1985.

[31] A. E. Brouwer and W. H. Haemers, Spectra of Graphs, Springer, New York, NY, USA, 2012.

[32] F. Zhang, Y.-C. Chen, and Z. Chen, "Clique-inserted-graphs and spectral dynamics of clique-inserting," Journal of Mathematical Analysis and Applications, vol. 349, no. 1, pp. 211-225, 2009. 
[33] A. K. Kelmans, "The properties of the characteristic polynomial of a graph," in Cybernetics-in the service of Communism, Vol. 4, pp. 27-41, Energija, Moscow, Russia, 1967. 


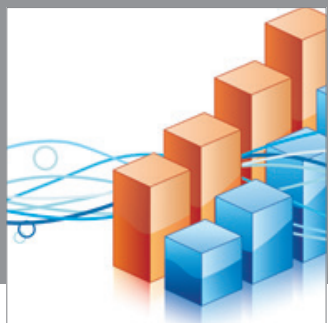

Advances in

Operations Research

mansans

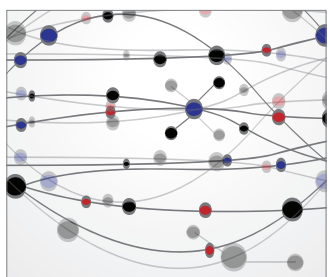

The Scientific World Journal
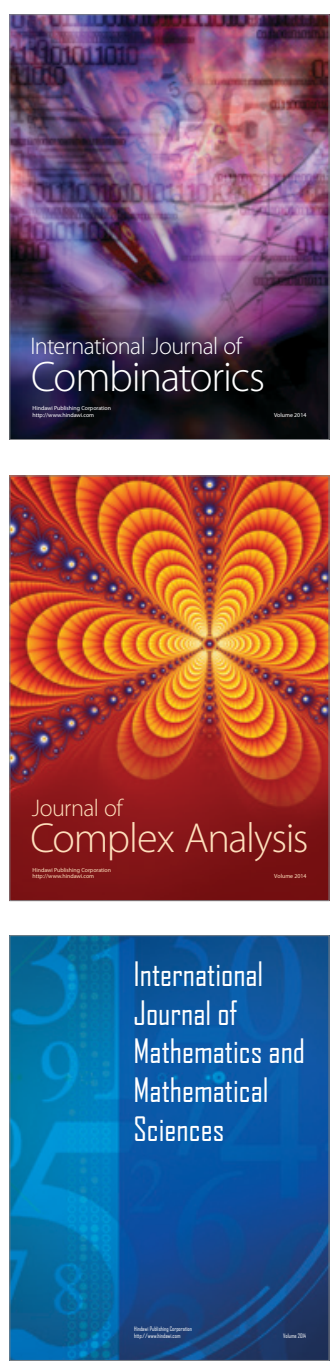
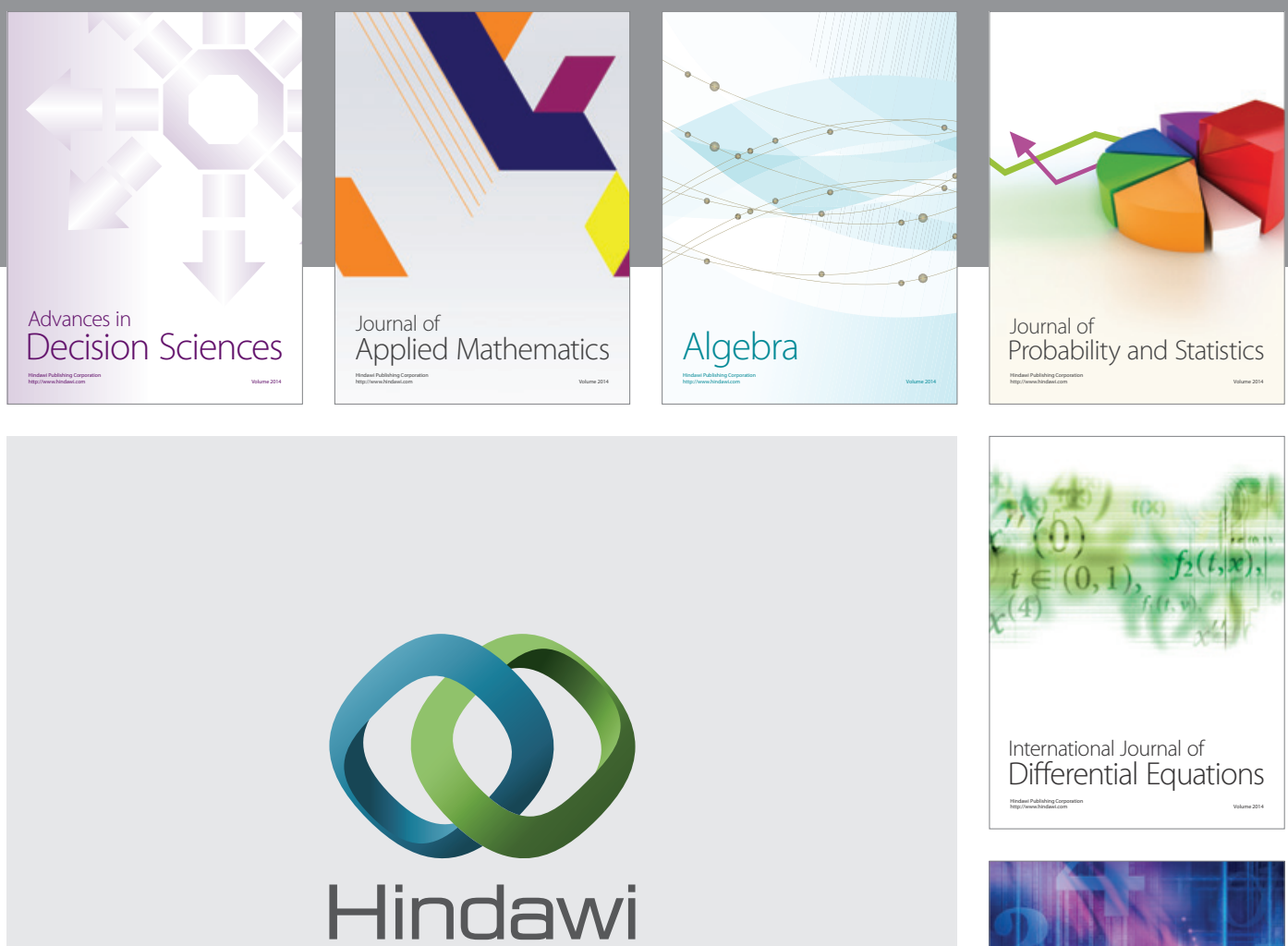

Submit your manuscripts at http://www.hindawi.com
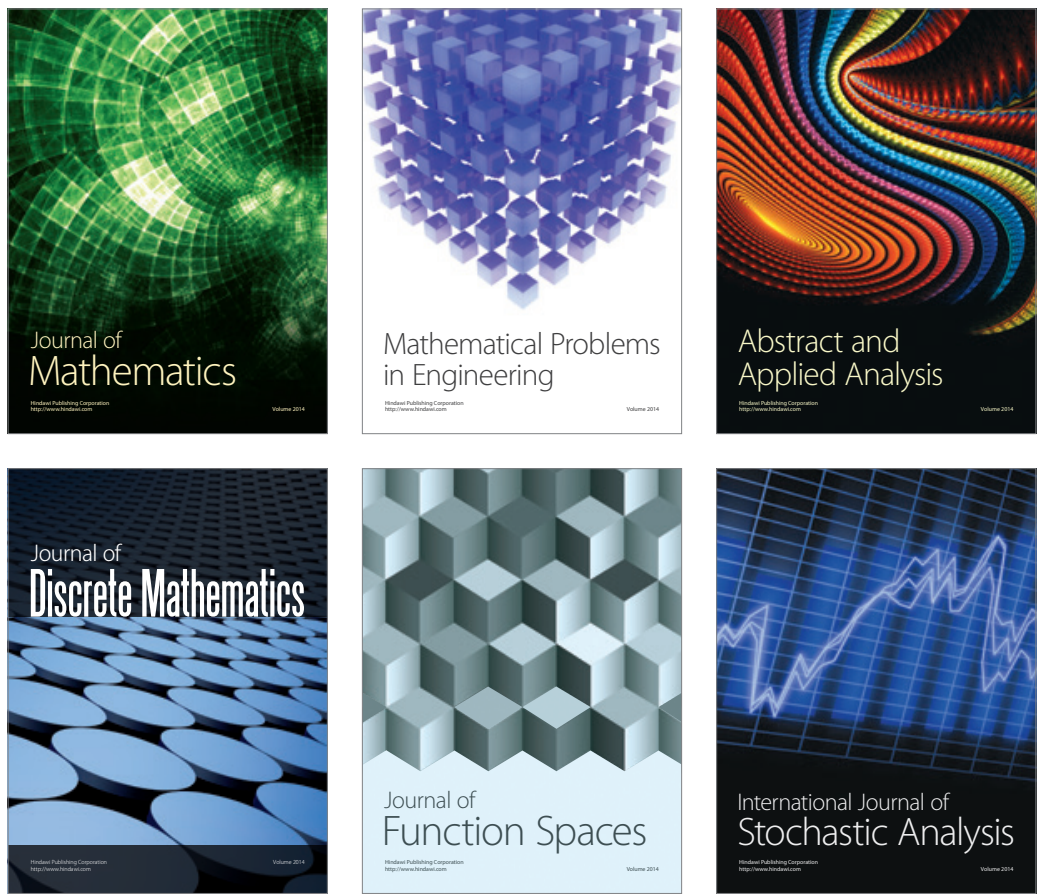

Journal of

Function Spaces

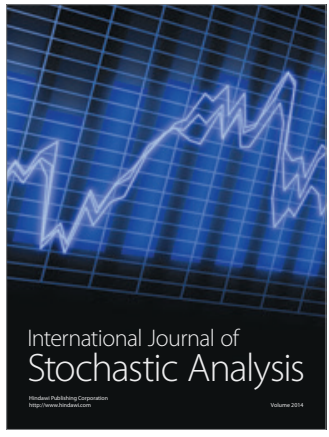

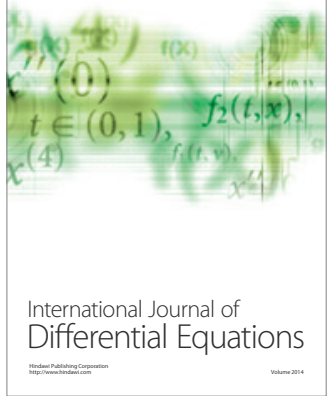
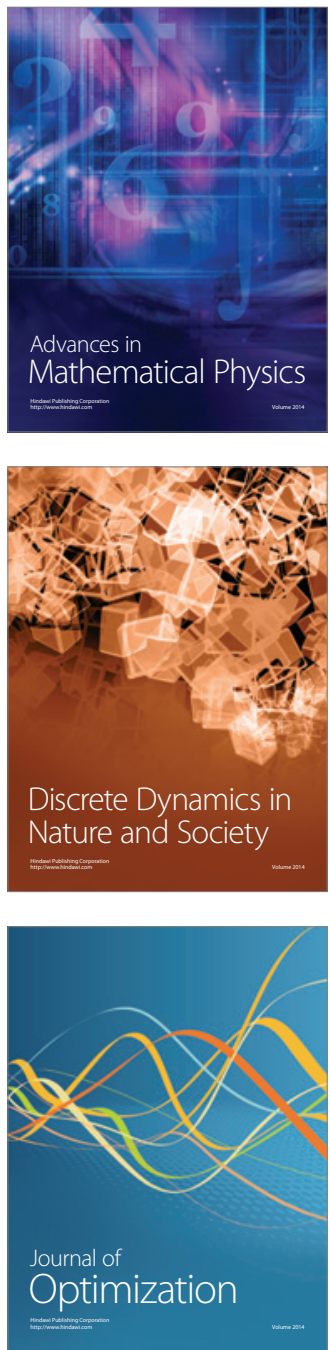